\title{
Wallmapu, terre de conflits et de réunification du peuple mapuche
}

Ana Guevara et Fabien Le Bonniec

\section{(2) OpenEdition}

\section{Journals}

Édition électronique

URL : https://journals.openedition.org/jsa/10631

DOI : 10.4000/jsa.10631

ISSN : 1957-7842

Éditeur

Société des américanistes

\section{Édition imprimée}

Date de publication : 20 décembre 2008

Pagination : 205-228

ISSN : 0037-9174

\section{Référence électronique}

Ana Guevara et Fabien Le Bonniec, «Wallmapu, terre de conflits et de réunification du peuple mapuche ", Journal de la Société des américanistes [En ligne], 94-2 | 2008, mis en ligne le 27 mai 2009, consulté le 03 septembre 2022. URL : http://journals.openedition.org/jsa/10631 ; DOI : https:// doi.org/10.4000/jsa. 10631 


\section{CHILI, ARGENTINE}

\section{WALLMAPU, TERRE DE CONFLITS ET DE RÉUNIFICATION DU PEUPLE MAPUCHE}

Les Mapuche ${ }^{1}$, anciennement appelés Araucans, font partie des populations les plus étudiées du continent sud-américain, ils ont fait l'objet d'un grand nombre de travaux depuis plus d'un siècle, que ce soit du côté chilien ou du côté argentin. Les recensements officiels, par ailleurs sujets à controverse, estiment à 600000 le nombre de personnes se considérant " appartenir à l'ethnie mapuche » au Chili, et à 113000 celles se reconnaissant appartenir ou être descendants « de première génération du peuple mapuche » en Argentine ${ }^{2}$. La résurgence, ces quinze dernières années, de leurs revendications sur les scènes publiques nationales et internationales a provoqué un regain d'intérêt pour l'histoire et la culture de ce peuple, engouement que l'on peut constater à travers la multiplication de publications, mais également de documentaires ou encore de sites internet.

Parallèlement à cette médiatisation, la réémergence du mouvement mapuche s'est illustrée par sa capacité à développer un discours, mais également des relations politiques, transandines, c'est-à-dire entre communautés et organisations des deux côtés de la Cordillère. Une telle velléité de déconstruire les frontières, notamment en s'attaquant aux mythes nationaux, affïchée par les Mapuche, n'a pas laissé indifférents les chercheurs qui s'intéressent à ces populations. De plus en plus, ces derniers ont dû prendre en compte, voire adopter, des catégories façonnées par les organisations et les historiens, anthropologues et autres professionnels indigènes dénommés « intellectuels mapuche », tant dans leur construction de l'objet d'étude que dans leurs analyses. Alors que, jusqu'à récemment, les traditions historiographiques nationales aidant, il était d'usage de respecter les frontières et de parler des Mapuche chiliens et des Mapuche argentins, de plus en plus de chercheurs optent pour essayer de faire une anthropologie et une histoire transandine de ce peuple qui, selon une rhétorique de plus en plus répandue, existait bien avant la formation des États nationaux. C'est ainsi que les terminologies Wallmapu («l'ensemble du territoire mapuche»), Puelmapu ( « terres à l'est », c'est-à-dire en Argentine), Ngulumapu (« terres de l'ouest », au Chili), façonnées ces dix dernières années par le mouvement mapuche, sont devenues courantes dans la littérature scientifique (Figure 1). Aussi nous est-il apparu naturel d'évoquer dans cette chronique la situation du peuple mapuche de 
cette même façon, en signalant les similitudes de contextes, de mobilisations et de réponses qui y sont données, tout comme ce qui les particularise, notamment du fait de l'existence de deux types d'État, l'un centralisé et l'autre fédéré, impliquant des politiques publiques différenciées.

\section{UNE HISTOIRE COMMUNE}

Notre choix de perspective, celui de considérer le peuple mapuche dans son ensemble et non pas comme deux réalités séparées de par leur situation géographique, se justifie ne serait-ce qu'aux yeux d'une histoire marquée par des échanges transandins incessants et ininterrompus depuis le $\mathrm{xvII}^{\mathrm{e}}$ siècle. Les Mapuche jouèrent un rôle central dans la circulation transandine de bétail, de peaux, de sel et de ponchos. L'adoption du cheval par les Indiens situés sur une frange restée indépendante au sud de la Capitainerie générale du Chili est considérée comme un moment important, car il va permettre à ceux-ci non seulement de résister aux Espagnols, mais également d'entreprendre des voyages transandins et d'aller se mélanger avec les populations vivant dans la Pampa argentine. Cette version de l'histoire, appelée " araucanisation de la Pampa », bien que partagée par un grand nombre d'historiens, est cependant rejetée par la plupart des organisations indigènes qui considèrent que les Mapuche étaient présents sur un large territoire allant de l'océan Pacifique à l'océan Atlantique bien avant l'arrivée des conquistadores. Nous le verrons, les enjeux contemporains de l'autochtonie au Chili et en Argentine sont devenus fondamentaux, notamment pour les luttes de légitimité territoriale, mais les critères utilisés pour définir l'« autochtonéité » se fondent souvent sur un essentialisme pourtant dépassé. L'histoire commune des populations mapuche d'Argentine et du Chili, les échanges de biens, les déplacements humains et les alliances politiques qui la caractérisent, ont contribué, de part et d'autre, à la construction de récits oraux marquant les imaginaires : au Chili, le « voyage en Puelmapu » et, en Argentine, « les narrations d'origines ».

\section{« RÉCITS DES ORIGINES » ET DES SOUFFRANCES, HISTOIRES DES FAMILLES}

Les « narrations d'origines » et les récits oraux des deux côtés de la Cordillère ont permis la construction d'une certaine subjectivité mapuche se fondant sur des histoires de familles aux origines communes. Les « narrations d'origines » relatent le passage de la Cordillère des ancêtres provenant de Ngulumapu comme un voyage initial et fondateur des communautés aujourd'hui situées en Argentine ${ }^{3}$. Ces narrations, généralement produites dans des contextes familiaux, se différencient des discours plus politiques dirigés vers l'extérieur qui légitiment leurs 


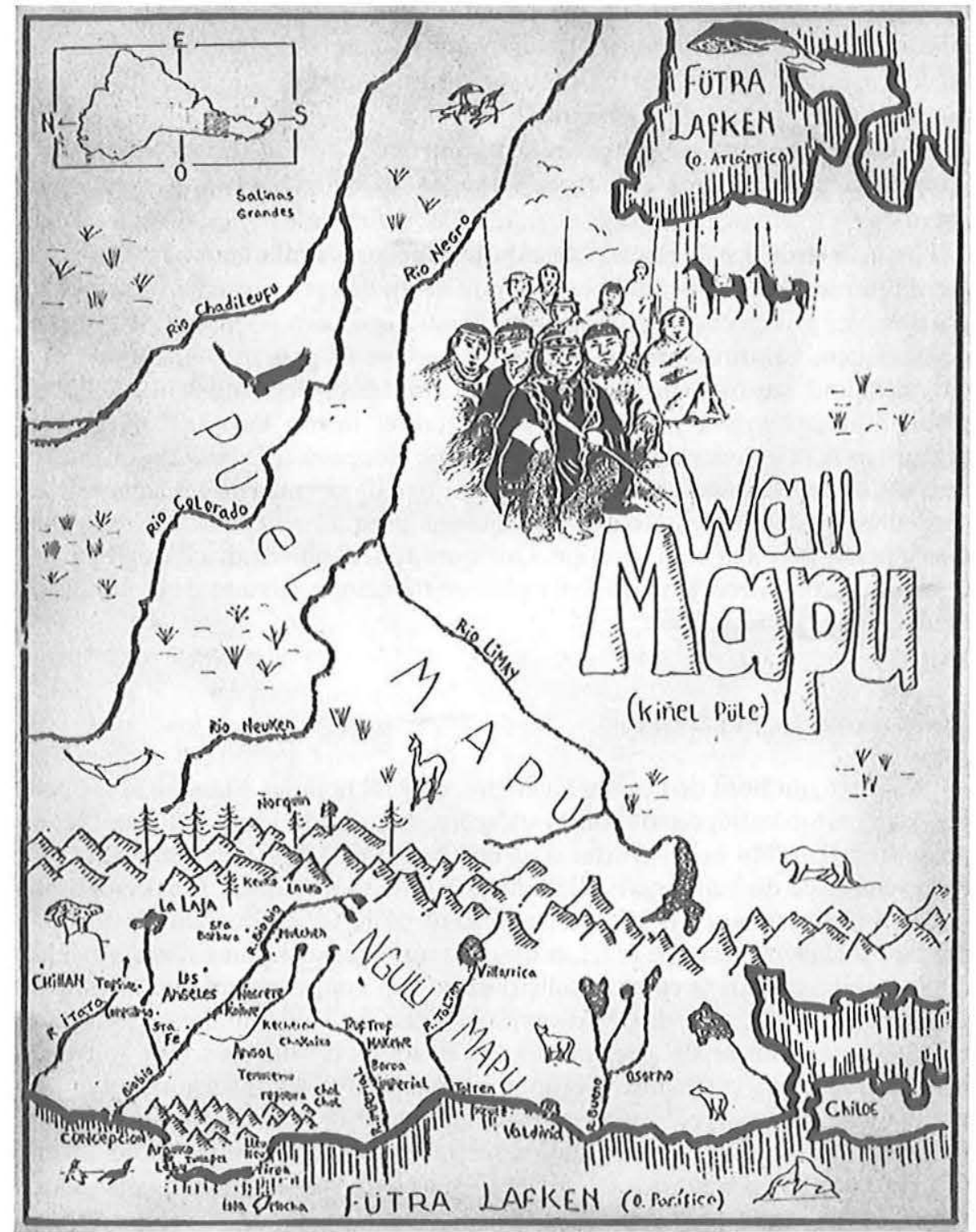

FIG. 1 - « Le territoire mapuche au moment des derniers Parlamentos et traités ( $\mathrm{xIX}^{\mathrm{e}}$ siècle) » (carte de Pablo Marimán, in Marimán Pablo (éd.), 2002, Parlamento y territorio mapuche, Instituto de Estudios Indígenas, Escaparate, Concepción, p. 54). 
revendications territoriales sur une supposée présence ancestrale des Mapuche en Puelmapu. D'autres récits de déplacements forcés rendent compte des discontinuités qui ont ponctué la mémoire des familles mapuche. Parmi ces récits, on rencontre celui de Felisa Curruhuinca, mapuche quadragénaire habitant la communauté Curruhuinca de Puelmapu.

Felisa se souvient avec une grande émotion de l'histoire de son grand-père qui illustre les déplacements de ce peuple entre les deux pays. Son grand-père, Amadeo Curruhuinca, alors âgé de douze ans, a dû fuir la région d'Azul (au sud de l'actuelle province de Buenos Aires) car sa propre famille était persécutée par les militaires argentins pendant la "Conquête du désert » aux environs de 1880. Il a donc été pris en charge par une autre famille qui l'a mené jusqu'à la province de Neuquén. Constitué de dix adultes, le groupe fit près de 900 kilomètres à cheval quand, au moment de traverser la Cordillère des Andes, il fut décidé d'abandonner l'enfant pour ne plus se retarder face à l'avancée de l'armée argentine. À ce moment-là, une jeune femme s'opposa à l'abandon et prévint l'enfant après avoir tenté de dissuader le groupe de commettre cet acte. Elle lui conseilla de suivre les empreintes des chevaux pour les retrouver... et c'est ainsi que le grand-père de Felisa survécut. Quelques années plus tard, à l'âge de 25 ans, il revint en Argentine, pays qu'il n'a plus quitté depuis, laissant de la famille de l'autre côté de la Cordillère.

\section{Les voyages en Puelmapu}

À Toltén, au bord de l'océan Pacifique, un vieil homme, Manuel, se rappelle des voyages qu'entreprenait son grand-père, Juan de la Cruz Pinchumilla, en Argentine. Il partait à cheval armé d'un revolver durant plusieurs semaines, avant de revenir avec du bétail qu'il allait ensuite vendre à Valdivia. C'est en grande partie de ce commerce transandin que Juan de la Cruz Pinchumilla tira son prestige d'ulmen, d'homme riche, et qu'il fut nommé par l'État chilien, au début du $\mathrm{xx}^{\mathrm{e}}$ siècle, chef de la communauté portant son nom. On peut ainsi retrouver; en arpentant les contrées de l'Araucanie chilienne, des récits similaires de voyages en Puelmapu. Sortes de négatifs des "narrations d'origines ", ces souvenirs mettent au jour les vastes réseaux toponymiques, familiaux et sociaux qui se sont tissés lors de ces expéditions commerciales. José Ancán, historien d'origine mapuche, s'est intéressé à la mémoire, souvent orale, de ces expéditions ${ }^{4}$. Il l'a fait perdurer en écrivant des articles et en repartant sur les traces de ces grands voyageurs. S'inspirant d'un récit recueilli au début $d u x^{e}$ siècle par le père capucin Ernesto Wilhelm de Moesbach, il a refait une partie du parcours décrit par Pascual Coña, un paysan mapuche qui vivait au bord du lac Budi, sur la côte Pacifique, et qui était parti accompagner son « cacique », Painemilla, à Buenos Aires. Le témoignage de Pascual Coña montre que ces voyages transandins ne 
répondaient pas seulement à des raisons d'ordre économique, mais donnaient également lieu à des relations diplomatiques, diverses pratiques d'alliances familiales, de rites d'initiation et de sémantisation des lieux et des territoires. Il décrit, par exemple, le cérémoniel de l'awun, consistant en une chevauchée en forme de cercle, qui fut organisé en leur honneur lorsqu'ils arrivèrent sur les terres d'Ancatrir, à San Junín dans l'actuelle province de Neuquén. Pascual Coña était étonné des grandes similitudes existant au niveau de la langue et des rituels entre sa communauté d'origine et les communautés de Puelmapu.

\section{Formation des États, PERTe de souveraineté ET fragmentation du WALLMAPU}

La constitution, de part et d'autre de la Cordillère des Andes, des États nationaux durant le $\mathrm{XIx}^{\mathrm{e}}$ siècle marqua la fin de cette indépendance et de cette libre circulation. Ce qu'on appela par euphémisme la « Pacification de l'Araucanie » et la "Campagne du désert » fut synonyme de véritables guerres envers les populations indiennes de ces territoires. Prises en étau entre les avancées des troupes argentines et chiliennes, beaucoup de familles durent être déplacées, leurs terres confisquées, leurs biens détruits et leurs troupeaux volés ou affamés. Cette violence "fondatrice» et cette perte de souveraineté politique, territoriale et économique, sont aujourd'hui d'autant plus prégnantes dans la mémoire des populations mapuche, en milieux urbain et rural, qu'elles sont relativement récentes au regard de l'histoire de ce peuple.

L'historien Walter Delrio qui a travaillé sur ces «mémoires de l'usurpation » n'hésite pas à parler, dans le cas de l'Argentine, d'un véritable génocide qui aurait été perpétré envers des Indiens qui furent regroupés dans des " camps de concentration ». Ces violences physiques et symboliques n'eurent pas la même intensité et ne s'appliquèrent pas toujours de la même manière au Chili et en Argentine, mais elles touchèrent la mémoire d'un peuple pour qui les frontières nationales n'avaient guère de sens, les logiques de lignages et d'alliances primant.

La constitution de la propriété australe, c'est-à-dire la mise en réserve des Indiens et surtout la colonisation d'une grande frange des territoires indigènes qui suivirent ces guerres, connut également ses variantes selon les pays, mais également selon les provinces d'Argentine. Ce sont différents corps législatifs qui furent mis en place pour imposer le système de communauté aux indigènes. Au Chili, cette politique se caractérisa principalement par la remise de titres communautaires - les títulos de merced - entre 1884 et 1929 et la réduction de $90 \%$ de leur territoire, principalement des plaines fertiles, impliquant le passage à un système productif de petit paysannat. Le gouvernement chilien encouragea la venue de colons nationaux et européens sur ces territoires ${ }^{5}$ afin de travailler des 
terres que, selon lui, les Indiens étaient incapables d'exploiter. Du côté argentin, ce processus est plus complexe et se distingue clairement de la situation chilienne du simple fait de l'existence d'une géographie, de paysages et d'une répartition démographique totalement différents. Par ailleurs, le mode d'organisation fédérale de l'Argentine a impliqué un traitement différencié des populations indigènes qui étaient installées dans plusieurs provinces. Certaines furent déportées pour former des colonies, tandis que d'autres obtinrent des titres de propriété sur des parcelles de terre qu'elles occupaient. Ce sont également les colons et les grandes entreprises de colonisation étrangères qui bénéficièrent de la plupart des terres indigènes. Que ce soit au Chili ou en Argentine, c'est dans cette violence constitutive des communautés contemporaines et dans la répartition inégale des terres que s'enracinent les origines des conflits actuels.

L'appauvrissement des Mapuche, ainsi que l'édification des frontières et des normes nationales, limitèrent les échanges transandins durant le $\mathrm{xx}^{\mathrm{e}}$ siècle, contribuant ainsi à séparer et distinguer, de part et d'autre de la Cordillère, des populations qui avaient pourtant des origines communes. Les migrations transandines ont cependant perduré, durant le $\mathrm{xx}^{\mathrm{c}}$ siècle, sous des formes plus oflicielles, pour des raisons d'ordre économique ou politique ${ }^{6}$.

\section{LE MOUVEMENT « PAN-MAPUCHE » CONTEMPORAIN}

Les premières organisations politiques mapuche du Chili (1910) et d'Argentine (au début des années 1970) ont, dans un premier temps, développé des discours ne prenant pas en compte la situation transandine de leur peuple, pour la simple raison qu'elles se dirigeaient aux responsables d'États distincts. Ce n'est que depuis ces vingt dernières années, dans un contexte marqué par l'internationalisation des luttes autochtones et la fin des dictatures, que les organisations mapuche du Chili et d'Argentine ont développé ensemble des stratégies, en remettant au jour leur histoire commune. L'un des exemples les plus significatifs est l'élaboration d'un drapeau de la nation mapuche, le wenu foye (Figure 2), aux débuts des années 1990, par une organisation située en Ngulumapu et qui est aujourd'hui couramment utilisé par les communautés d'Argentine. Nombreux sont aujourd'hui les échanges entre les organisations, certaines coordinations se revendiquant " pan-mapuche " ${ }^{7}$; des journaux mapuche n'hésitent pas à effacer les frontières sur les cartes oflicielles reproduites dans leurs colonnes pour représenter l'intégralité de leur territoire ${ }^{8}$.

Un exemple tout aussi significatif et récent de la vitalité des contacts transandins, chez le Peuple Mapuche, est l'organisation au début de l'année 2007 du Parlamento de Koz Koz réunissant environ 3500 personnes provenant des différents points du Wallmapu, de Buenos Aires à Bariloche en Puelmapu et de Valparaiso à Aysén en Ngulumapu. 


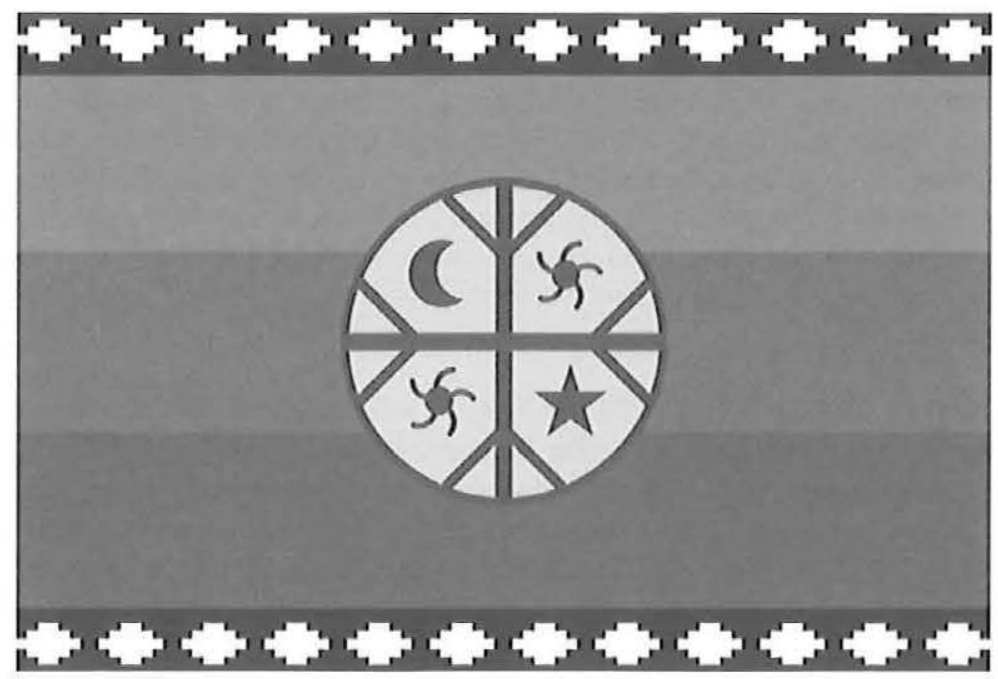

Fig. 2 - Wenu foye, drapeau mapuche.

Le lonko (chef) de la communauté Curruhuinca, Ariel Epulef, a fait partie des invités de Puelmapu à Koz Koz. Cet événement l'a marqué et a pris beaucoup d'importance dans sa vie de dirigeant, ne serait-ce que parce qu'il traversait pour la première fois la Cordillère des Andes. Avec sa soif de savoir et sa quête de la culture mapuche "originale », il a préparé ce voyage comme un retour aux sources. Pendant le Parlamento, le jeune lonko en profita pour discuter pendant des heures avec les personnes plus âgées, porteuses du kimun ("la sagesse »). Ainsi, il a pu répondre à ses multiples questions concernant la philosophie et les pratiques culturelles, tel le rituel du nguillatún qui l'impressionna par son apparente authenticité.

\section{Les PARLAMENTOS, 1907-2007}

La cérémonie traditionnelle, qui dura quatre jours, eut lieu là même où s'était déroulé le Parlamento de $\mathrm{Coz} \mathrm{Coz}{ }^{9}$ un siècle auparavant. Le Parlamento qui eut lieu en janvier 1907 est considéré comme la rencontre la plus importante ayant été réalisée après la "pacification » des Mapuche au Chili. La persistance de cet événement dans la mémoire sociale et sa commémoration contemporaine reposent plus sur la chronique éditée à plusieurs reprises d'un journaliste présent au Parlamento, Aurelio Díaz Meza, que sur la tradition orale. 
L'objectif du Parlamento était principalement d'améliorer la communication entre les caciques et de discuter des infortunes dont ils souffrent ; pour se raconter en famille, si on peut le dire ainsi, les atrocités inédites que les « Espagnols » commettent contre eux, écouter l'opinion des anciens, auxquels ils prêtent un grand respect et résoudre de façon commune ce qui serait opportun de faire pour sauver ce qui leur reste de leur patrie auparavant libre : leur terre, leur ruca et leurs animaux. L'hôte et organisateur de ce Parlamento, Manuel Curipangui-Treulen, cacique principal de Coz-Coz, est un vrai type d'Araucan. Grand, corpulent, d'aspect féroce, vif d'esprit et pensif. (Traduction des auteurs) ${ }^{10}$

La description que fit Aurelio Díaz de ce grand rassemblement met en évidence tout un rituel pour accueillir les invités, puis les sacrifices d'animaux, les danses, awun, les prières, les repas et les discours qui rythment la journée. C'est en effet en marge de ces rituels que les caciques des différentes zones faisaient preuve de leur habilité d'orateur, si essentielle pour la fonction qu'ils occupent. Leurs récits racontaient les injustices et les usurpations dont ils avaient souffert. On y retrouvait de nombreuses redondances, que ce soit au niveau des noms des usurpateurs comme des " techniques employées " : ruca ${ }^{11}$ incendiées, menaces, meurtres, faux contrats de vente, complicité des agents de l'État ou encore abus de confiance... Un consensus clair se dégageait : il fallait nommer un cacique représentant toutes les tribus pour aller à Santiago parlementer avec le Président, ouvrant un nouveau cycle de discussions pour nommer la personne la plus apte à ce rôle.

Un siècle plus tard, les différents voyages de caciques et dirigeants à Santiago, instaurés comme une tradition dans le mouvement mapuche, ne semblent pas avoir porté leurs fruits. Certains conflits évoqués en 1907 persistent, la mémoire perdure et les usurpateurs sont toujours présents. Ceux-ci ont changé de noms, mais également de « techniques ». Le Parlamento de Koz Koz célébré en janvier 2007, bien que se revendiquant avant tout comme un espace d'union et de spiritualité, n'échappa pas aux discussions sur ces nouveaux usurpateurs. Plus qu'un espace cérémoniel ou de revendication politique, le Parlamento de $\mathrm{Koz} \mathrm{Koz}$ s'est avéré être un véritable producteur de ce que l'on peut appeler une « subjectivité mapuche ", c'est-à-dire que cet événement, en devenant une référence commune autant pour les personnes présentes que pour l'ensemble du mouvement mapuche contemporain, a contribué à reformuler une identification et un sentiment d'appartenance à un même peuple.

La préoccupation des organisateurs pour réaliser une cérémonie respectant au plus près la tradition eut ses effets, marquant les esprits de nombreuses personnes, et notamment celles venues en quête d'authenticité. Parmi celles-ci, on retrouve des jeunes rappeurs mapuche des banlieues de Santiago, ou des familles de communautés de Puelmapu, tous ayant en commun d'avoir été stigmatisés durant de nombreuses années, comme des Mapuche acculturés du fait de leur condition d'exilés. Le purisme de la cérémonie constitue ainsi pour eux une forme 
de retour aux sources, une référence à un passé mythique. L'efficacité symbolique d'une telle cérémonie vient s'ajouter aux diverses pratiques relatives à l'usage du passé auxquelles ont recours aujourd'hui les Mapuche. Celles-ci s'inscrivent dans un travail permanent de relecture et de « reconstruction » d'une histoire qui, jusqu’à récemment, n'avait été écrite que par les vainqueurs. La chronique d'Aurelio Díaz fut non seulement utilisée pour la commémoration du centenaire du Parlamento de $\mathrm{Coz} \mathrm{Coz}$, mais également par des historiens qui la citent régulièrement pour montrer en quoi ce type de rassemblement constituait à lui seul une forme d'institution sociopolitique pour le peuple mapuche avant la perte de son indépendance ${ }^{12}$.

\section{LES NOUVEAUX USURPATEURS}

Le dernier jour du Parlamento, avant que tous les participants repartent vers leurs foyers respectifs, une déclaration est lue par Olga Curipán, une dirigeante de Buenos Aires qui n'est autre que la petite-fille du cacique Manuel CuripanguiTreulen qui, un siècle auparavant, avait été convoqué au Parlamento de Coz Coz. Debout sur une scène aménagée pour l'occasion, un micro pour amplifier sa voix, elle lit les conclusions, les stratégies retenues, les objectifs et les revendications qui y sont associées. Parmi celles-ci, l'exigence d'en terminer avec les « mégaprojets » en Wallmapu, rappelant que ce qui a tant été médiatisé sous le nom de conflit mapuche réside dans des situations concrètes où entrent en jeu des relations de pouvoir entre communautés, différentes instances de l'État et des intérêts privés.

Il existe ainsi un consensus, parmi les organisations mapuche de toutes tendances, pour pointer du doigt les multinationales qui envahissent leur territoire avec l'aval, voire le soutien, d'États inféodés au néolibéralisme. Un tel discours s'inscrit dans une rhétorique que l'on a vu émerger ces quinze dernières années sur les nouvelles formes de domination et d'exclusion provoquées par l'avènement de politiques néolibérales dans la plupart des pays d'Amérique latine. Et pour cause, la dictature chilienne de Pinochet et le "ménémisme » en Argentine, considérés comme les laboratoires du néolibéralisme en Amérique latine, ont produit des effets de déclassement des régions agricoles au profit des centres de pouvoir économique ${ }^{13}$. Le développement de ces pays s'est fait au détriment de l'environnement et des conditions sociales d'une grande partie de leur population. Une des régions formant partie du Wallmapu, l'Araucanie, a ainsi connu un important développement économique et démographique tout en rassemblant les indices de pauvreté les plus forts du pays.

Terreau fertile de conflits d'ordre écologique et social, les régions et les provinces du Sud du Chili et de l'Argentine sont aujourd'hui le théâtre de nouvelles mobilisations et stratégies d'alliance entre différents secteurs de la société civile. Les organisations et communautés mapuche montrent ainsi leur 
capacité d'innovation et leur ouverture, alors que beaucoup de leurs contradicteurs ont souvent essayé de les disqualifier en traitant leurs revendications de sectaires et de conservatrices, ou encore en essayant de les opposer aux autres secteurs de la société civile. Dans de nombreux cas, tels que la construction de barrages hydroélectriques ou d'usines polluantes, les Mapuche ne sont pas les seules populations affectées. Ils constituent souvent une avant-garde des résistances avant d'être ralliés par d'autres secteurs de la société civile et les mouvements écologistes et sociaux pour faire face aux nouveaux usurpateurs de leurs territoires (Figure 3).

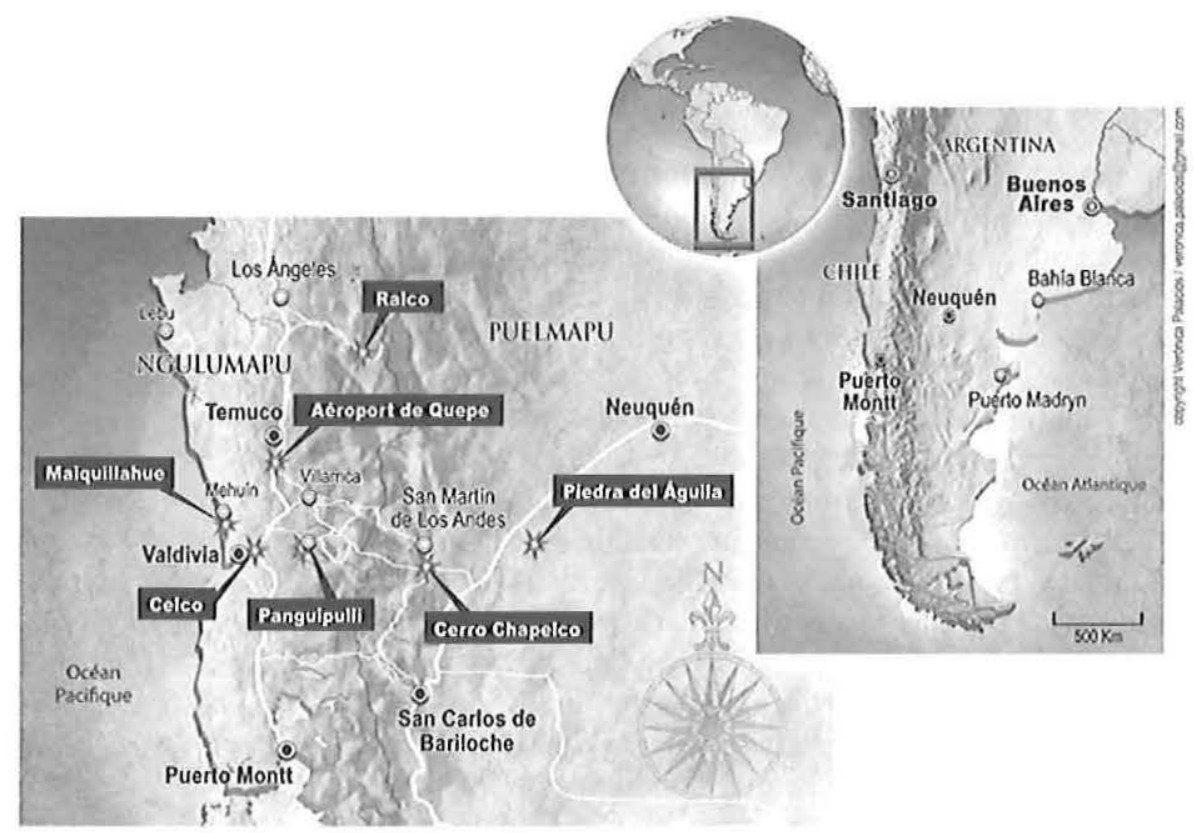

FIG. 3 - Situation géographique des conflits et des lieux mentionnés (carte conçue par Verónica Palacios, d'après les informations des auteurs).

\section{LA RADIO WAJZUGUN : LA VOIE INTERCULTURELLE}

Le cas de la radio Wajzugun ${ }^{14}$ (« la voix qui circule »), à San Martín de los Andes, dans la province argentine de Neuquén, est une illustration de l'alliance entre Mapuche et non-Mapuche dans une lutte commune. Le projet de la radio est né de l'ancienne radio associative FM Pocahuyo fondée au début des années 
1990 et qui n'avait qu'une fréquence locale. Grâce à l'appui et au travail en équipe entre diverses organisations de la société civile, le projet Wajzugun commence à se concrétiser et est suivi par plusieurs communautés mapuche qui s'inscrivent dans une démarche interculturelle et communautaire. Pour bien mener ce projet, des ressources financières ont été demandées par le biais de la Confederación Mapuche Neuquina à la Banque mondiale qui lui a versé les fonds via le ministère du Développement social. Parmi les cinq organisations qui intègrent la radio, on compte trois organisations mapuche.

Roberto, le locuteur principal de cette radio, est d'origine métisse et de parents chiliens, il est issu d'un quartier périphérique de San Martín, né d'une occupation illégale d'une partie du parc national Lanín. Il habite toujours ce quartier où se mêlent des Mapuche provenant de zones rurales proches et des émigrés chiliens, venus depuis les années 1940 pour travailler dans l'industrie du bois alors en pleine expansion. Soucieux de la perpétuation de la radio, Roberto a formé une équipe de jeunes Mapuche afin qu'ils créent leur propre programme. On peut ainsi entendre sur les ondes argentines de Wajzugun de la poésie et du rap mapuche provenant de Ngulumapu et des banlieues de la capitale chilienne. On peut en effet remarquer un renouveau culturel et artistique s'exprimant par l'apparition d'artistes mapuche de part et d'autre du Wallmapu faisant preuve d'innovation dans des domaines aussi différents que la sculpture, la musique, la poésie et le théâtre. Leurs créations s'inspirent autant de la tradition que d'influences venant de leur environnement urbain, rompant ainsi avec l'image folklorique et figée de la culture mapuche.

Wajzugun a permis de faire entendre une voix indienne et de rendre visibles la culture des communautés mapuche, leurs revendications actuelles et leurs attentes. La radio a renforcé les liens avec les Mapuche de Ngulumapu, offrant ainsi un lieu de rencontres et d'échanges transandins. La radio a permis aussi à des voisins, de cultures différentes, de s'organiser et de créer des liens sociaux avec les Mapuche à propos de problèmes communs qui les préoccupent. Le fruit de cette union est notamment la dénonciation du conflit lié au Cerro Chapelco, mais également la lutte contre les exploitations pétrolières et les projets de barrages hydroélectriques.

\section{Le conflit de Cerro Chapelco ou la remise en Cause d'un modèle de DÉVELOPPEMENT}

Depuis la fin des années 1990, les communautés mapuche Curruhuinca et Vera sont en conflit avec les exploitants de la station de sports d'hiver Cerro Chapelco, ainsi qu'avec le gouvernement provincial qui accorde la concession en échange d'un pourcentage des profits. Jusqu'à présent, cette concession a toujours été renouvelée sans que les autorités mapuche soient consultées. Les com- 
munautés concernées ${ }^{15}$ se trouvent entre 4 et $7 \mathrm{~km}$ de San Martín de los Andes, ville à forte croissance touristique.

Jusqu'en 1950, la ville de San Martín connaissait des échanges commerciaux importants avec le Chili et une économie de subsistance fondée sur les cultures maraîchères et l'élevage, ainsi que des petites scieries. Paradoxalement, les terres indigènes, " oubliées » pendant des décennies car de peu d'intérêt sur le plan économique, ont commencé, à la fin des années 1970, à faire l'objet de nouvelles et nombreuses convoitises. Aujourd'hui, ces terres sont perçues comme ayant une haute valeur paysagère et donc immobilière. En effet, elles se trouvent principalement autour du lac Lácar et au pied du Cerro Chapelco, au cœur de l'une des plus grandes stations de sports d'hiver d'Argentine.

En raison des énormes richesses naturelles présentes dans les alentours du Chapelco, il existe de nombreuses frictions entre ceux qui veulent accéder à ces ressources (et les exploiter) et les populations autochtones. Les communautés mapuche demandent une délimitation visible de leurs terres situées autour du Cerro. Cette exigence est essentielle dans la construction d'un espace territorial propre, tant au niveau politique que symbolique. Les communautés cherchent ainsi à avoir une place dans la gestion d'un vrai projet de développement durable lié à une reconnaissance de leur culture.

Les communautés doivent faire face à de nouvelles nuisances telles que le bruit, la construction de routes sur leur territoire ou la contamination de l'eau, produisant des confrontations tout au long de l'année. Durant l'été, le problème se pose pour les éleveurs qui partent en transhumance ; d'année en année, ils voient leur espace de pâturage de plus en plus restreint et de plus en plus éloigné. En hiver, alors que la saison touristique bat son plein, le problème, le plus grave sans doute, qui se pose est celui de la surexploitation des ressources en eau. Les activités humaines génèrent aussi une masse de déchets organiques et non organiques qui provoque une pollution des eaux. Depuis plusieurs années, les déchets sont rejetés dans la rivière sans avoir été traités au préalable. Les habitants des communautés qui se trouvent en aval et qui puisent l'eau directement à la rivière consomment donc une eau contaminée, tandis que la station Chapelco, située en amont, profite, elle, de l'eau pure venant des montagnes. De plus, en période estivale, quand les pluies deviennent rares, une partie de la communauté Curruhuinca est totalement privée d'eau. N'ayant jamais connu dans le passé ce type de pénurie, les habitants n'ont jamais mis en place de pratiques de stockage d'eau et sont désemparés.

Caractérisé par la forte présence de rivières et de lacs, l'environnement de Chapelco a suscité le développement de tout un imaginaire, une mémoire sociale liée à l'eau, dont témoigne avec une grande richesse la tradition orale des populations locales. Les agressions continuelles du milieu naturel dues à l'exploitation touristique ont contribué à la transformation des pratiques culturelles concernant le rapport à l'eau et la relation avec la nature. Le Cerro Chapelco est 
une montagne importante dans la conception du monde mapuche. Habitée par un ngen, une force de la nature, on lui voue un immense respect et l'on ne manque pas de lui demander la permission avant d'entrer dans son espace. Les entreprises touristiques portent bien évidemment un regard complètement différent sur le territoire de Chapelco, où la population mapuche ajoute au lieu sa touche folklorique et, surtout, fournit une main d'œuvre disponible et bon marché pour tous les services d'hôtellerie et de restauration.

L'incapacité des autorités provinciales et de la justice pour reconnaître et résoudre ces divers conflits provoqués par l'implantation de la station de ski ont amené les habitants des communautés Curruhuinca et Vera à réagir. Ils organisent régulièrement des manifestations et des barrages routiers pour dénoncer cette situation et tenter de la médiatiser, ainsi que pour faire prendre conscience aux touristes des effets négatifs de leur présence. Les négociations et la possibilité de déboucher sur des accords se voient d'autant plus limitées qu'il existe des positions divergentes à l'intérieur même des communautés du fait des enjeux sous-jacents au conflit. C'est notamment le problème qui s'est posé lors de l'accord récemment signé entre l'État provincial, la municipalité de San Martín et l'une des deux communautés en conflit ; celle-ci a obtenu la reconnaissance des " limites historiques» de son territoire ainsi que d'importantes compensations économiques échelonnées sur trente années. Ce même accord qui avait été proposé à la communauté Curruhuinca fut refusé par ses représentants, estimant qu'il ne répondait pas à leur aspiration de co-gérer l'ensemble du territoire de Chapelco en vue de mettre en place un autre système de développement.

On le voit à travers cet exemple, le développement du tourisme, que ce soit dans la Patagonie argentine ou dans le sud du Chili, a signifié pour une partie de la population mapuche qui y vit une aubaine pour améliorer sa situation économique, mais au prix d'importants impacts. Ces projets menacent non seulement l'environnement naturel et spirituel d'une majorité des communautés, mais également l'existence même de leurs habitants. Par exemple, la région de l'Araucanie est devenue un lieu de passage, voire une destination, obligatoire pour les touristes, obligeant à développer de grands axes routiers et des aéroports à proximité des communautés. Il y a quelques années, le ngenpin (" Maître de la parole » et des cérémonies traditionnelles), Jorge Pichiñual, récemment disparu, avait réussi à organiser un front commun parmi les communautés du lac Budi, contre la construction d'une autoroute qui était censée amener les touristes au bas de leur porte. Les autorités régionales qui faisaient la promotion de ce projet s'offusquèrent de cette fervente opposition des communautés à leur propre développement, mais Jorge et une cinquantaine de personnes se dressèrent physiquement contre la construction de cette autoroute créatrice de plus d'impacts négatifs que d'avantages : passage à proximité des lieux de cérémonies, destruction du paysage de l'unique lac salé d'Amérique latine, mise en péril des enfants et des animaux aux abords de la route. Le succès de cette résistance a certainement résidé dans la 
capacité de ses dirigeants à lier un discours « cosmovisionniste » et politique avec des préoccupations plus pratiques.

\section{L'AÉroport DE QUEPE OU LE SIMULACRE DE LA CONSULTATION CITOYENNE}

Plus récemment, c'est la construction d'un aéroport international à Quepe, à $14 \mathrm{~km}$ de la capitale régionale de Temuco, qui a provoqué une forte opposition d'une dizaine de communautés des alentours. Cette nouvelle infrastructure doit remplacer l'aéroport de Maquehue, implanté depuis une soixantaine d'années en plein milieu de communautés mapuche. Cette fois-ci, les communautés, qui ont créé une association, comptent faire entendre leur opposition à un projet pour lequel elles n'ont pas été consultées. Les institutions de l'État promotrices de l'aéroport pensaient que les habitants n'y verraient aucun inconvénient puisque le site choisi n'était pas situé sur des terres indigènes et que des subventions avaient été promises aux communautés adjacentes en échange de leur consentement. Mais, pour ces dernières, c'est leur espace socio-territorial, différent de la conception étatique et fragmentaire des territoires indigènes, qui serait agressé par l'infrastructure aéroportuaire : il a ainsi été demandé d'interdire les vols les jours de cérémonie.

Autre sujet de préoccupation, la pollution que la construction de l'aéroport entraînera sur les terres voisines où vivent des petits paysans chiliens et des communautés indigènes dont la production agricole et laitière est réputée pour sa qualité. L'avocat mapuche Richard Caifal, membre de l'association, explique que :

Les communautés ne sont pas contre la construction d'un aéroport. Ce que nous questionnons c'est l'emplacement actuel choisi par les autorités et les mécanismes peu démocratiques qui ont été mis en œuvre. Nous n'avons pas une position hostile au développement, ni même contre l'aéroport, ce que nous demandons c'est que ce mégaprojet soit transféré vers un autre secteur et que l'on respecte une fois pour toutes nos droits. ${ }^{16}$

Les enjeux soulevés par cette opposition à la construction d'un aéroport relèvent ainsi de la sphère démocratique dans un pays en permanente " transition démocratique ", c'est le fonctionnement même de l'État qui est mis en cause par les communautés. Leurs dirigeants suivent toutes les procédures légales auprès des tribunaux et des institutions publiques. Cela ne les empêche pourtant pas de pointer le simulacre de consultation citoyenne, les incohérences et les vides juridiques de lois censées protéger l'environnement et les populations indigènes. Ils considèrent que, dans la pratique, celles-ci sont rédigées pour faciliter, voire encourager, les « mégaprojets ». Cette dernière conclusion a notamment été faite par l'anthropologue Roberto Morales qui, après avoir rédigé des études d'impact 
pour la Commission Nationale de l'Environnement (CONAMA), ainsi que le prévoit la loi, a vu les projets correspondants acceptés malgré les nombreuses critiques qu'il avait pu émettre.

\section{LE BARRAGE DE RALCO : CHRONIQUE D'UN ETHNOCIDE ANNONCÉ}

Ces incohérences, nous les avons vues à l'œuvre il y a plusieurs années dans le cas de la construction du barrage hydroélectrique de Ralco, dans la Cordillère de l'Alto Bío Bío (VIII région du Chili), sur les territoires de populations mapuchepehuenche. Comme il était prévu par la «Loi environnementale », promulguée au retour à la démocratie en 1994, le même Roberto Morales avait participé à l'évaluation de l'étude d'impact environnemental. Ce travail fut commandité par l'entreprise d'électricité Endesa pour construire un barrage hydroélectrique d'une puissance de 570 mégawatts, provoquant l'inondation de plus de 3000 hectares de terres dont certaines appartenaient à des communautés. Outre l'inondation de plusieurs anciens cimetières ainsi que de terrains de cérémonies, Roberto Morales avait pointé la série d'impacts négatifs provoqués par le déplacement des familles pehuenche sur des terres où elles ne pourraient plus pratiquer leur mode de vie traditionnel ${ }^{17}$. Pourtant, dès 1997, le gouvernement d'Eduardo Frei (1994-2000) autorisa la construction du barrage, et cela, malgré l'avis négatif d'un certain nombre d'experts et l'opposition de plusieurs familles pehuenche, dont les sœurs Quintremán, efligies de la résistance au projet de la multinationale Endesa.

La Loi indigène, promulguée en 1993, était censée empêcher tout type de déplacement de populations sans le consentement préalable de celles-ci. Mais le gouvernement d'Eduardo Frei et l'entreprise Endesa réussirent à passer outre cette obligation au prix de plusieurs mois de procédures juridiques. Allant jusqu'au palais présidentiel et aux tribunaux à Santiago, au Congrès à Valparaiso, participant à des blocages de routes, manifestant dans les rues des différentes villes, les sœurs Quintremán essayèrent par tous les moyens de protéger « leur terre-mère ", mais les tractations et fortes pressions politiques et économiques eurent raison de leur attachement à leur territoire ancestral. Alors que la construction du barrage était sur le point d'être terminée, la Cour interaméricaine des Droits de l'Homme, à laquelle avaient eu recours les dernières familles pehuenche refusant tout déplacement, trancha en faveur de ces dernières. Le verdict imposa un accord à l'amiable signé entre l'État chilien, Endesa et les Pehuenche. Celui-ci, scellé en 2003, engage l'entreprise Endesa à verser d'importantes indemnités, à assurer une assistance en matière de développement pour les familles déplacées, ainsi qu'à les doter de terrains situés à une vingtaine de kilomètres de leur communauté d'origine. L'État chilien, quant à lui, promet la mise en place de différents plans d'assistance dans la zone de l'Alto Bío Bío, ainsi que la création d'une commune pehuenche réunissant les habitants des communautés 
vivant dans la Cordillère, mais aussi la reconnaissance constitutionnelle des peuples indigènes et la ratification de la Convention 169 de l'Organisation internationale du Travail.

Le cas de Ralco est emblématique à plus d'un titre : il a montré la capacité des organisations mapuche soutenues par les écologistes à recourir à des instances internationales afin de contredire les décisions prises par les tribunaux nationaux ; il représente également la première opposition des communautés mapuche à la construction de barrages hydroélectriques qui, aujourd'hui, se multiplient dans toute la Patagonie.

\section{Barrage de Piedra del Águila ou la redécouverte des grottes sacrées}

De l'autre côté de la Cordillère, la construction d'un barrage situé à $230 \mathrm{~km}$ de la ville de Neuquén, en Argentine, sur le fleuve Limay, a provoqué le déplacement forcé des habitants de la communauté Ancatruz. Ce barrage, du nom de « Piedra del Águila », le plus grand d'Argentine, d'une puissance de 1400 megawats et d'une longueur de $800 \mathrm{~m}$, a été construit par l'entreprise multinationale Hidronor S.A. et commença à fonctionner au milieu des années 1990.

Comme l'explique l'anthropologue Juan Carlos Radovich qui a suivi le projet depuis ses origines: " Au début, le barrage Piedra del Águila fut perçu par la population touchée comme un projet de l'Etat argentin qui avait pour objectif de promouvoir le développement régional. Dans un premier temps, le barrage fut "idéalisé" par les locaux et conçu temporellement de manière diffuse " ${ }^{18}$. Au fil des années, face à l'immensité de ce projet, à l'afflux de travailleurs, de machines lourdes, les dégâts sur l'environnement et les changements radicaux du paysage, la perception des habitants a changé et ils se sont rendu compte que ce projet pharaonique produirait des conséquences négatives pour eux, dans l'avenir.

Il y avait à l'époque un vide juridique concernant la protection du territoire et de la culture mapuche, car la reconnaissance constitutionnelle ne fut promulguée qu'en 1994. Oscar Curruhuinca, dirigeant mapuche, a travaillé pendant quelques années dans cette énorme entreprise. Il a été impressionné par les dégâts causés à la nature, mais ce qui l'a le plus marqué, c'est la découverte qu'il fit en participant aux excavations des fondations du barrage, chantier qui a duré environ 7 ans : " On a trouvé des fleuves souterrains asséchés à partir de $70 \mathrm{~m}$ de profondeur comme nos anciens nous l'avaient racontés, sortes de tunnel où pouvaient tenir 6 à 7 personnes. C'est à ce moment-là que je me suis rendu compte que ces histoires des anciens étaient possibles ». Les fleuves asséchés décrits par Oscar ont une grande signification dans la tradition orale des communautés de la Cordillère et d'une partie de la côte Pacifique. En effet, les tafu ou weniï, sorte de "grottes sacrées », étaient utilisés autrefois par les Mapuche, d’après leur mémoire sociale, 
soit pour communiquer entre le Chili et l'Argentine, soit pour entrer en contact avec les ancêtres ou encore pour s'y réfugier.

La construction de barrages dans le Sud du Chili et de l'Argentine reste encore d'actualité, et l'on a pu assister ces dernières années à une opposition des communautés ou des populations locales affectées ainsi que des organisations écologistes soutenues notamment par des philanthropes étrangers. Une campagne chilienne, "Patagonie sans barrage " ${ }^{19}$, lancée en 2007 a pris une grande ampleur avec la venue de stars hollywoodiennes afin de protéger ce qui est présenté comme l'un des derniers réservoirs d'eau pure de la planète. La Patagonie est ainsi devenue un espace physique et symbolique où se reconfigurent des luttes communes dépassant toutes sortes de barrières (ethniques, nationales, économiques, politiques). Cette région qui, durant longtemps, fut reléguée aux confins du monde se révèle être dans l'actualité source d'enjeux géopolitiques considérables eu égard aux convoitises existant autour de la gestion de ses ressources naturelles.

\section{L'EXPLOITATION FORESTIÈRE EN WALLMAPU ET SES MAUVAIS AUGURES}

L'exploitation du bois par de grands consortiums constitue aujourd'hui un nouveau problème auquel les communautés mapuche doivent faire face. Les vastes forêts primaires de la Patagonie ont, depuis leur découverte, suscité l'intérêt des Espagnols, puis des colons étrangers au $\mathrm{xx}^{\mathrm{e}}$ siècle. Ceux-ci étaient venus établir des scieries ainsi que de grandes propriétés destinées à l'agriculture et à l'élevage sur les terres dépouillées de leurs bois. Mais c'est à la fin des années 1970 que l'État chilien intensifie sa politique de développement forestier commencée dans les années 1930, dans le sud du pays, avec l'apparition de multinationales à capitaux étrangers et nationaux.

Avec les logiques du développement économique néolibéral actuel, on se demande quelles sont les politiques viables de protection de la nature et de développement durable. L'État favorise les exploitations de bois dits " exotiques », comme le pin et l'eucalyptus, en subventionnant leur plantation jusqu'à $90 \%$ de l'investissement nécessaire. En dehors du Wallmapu, ces plantations se font dans des zones soumises à la disparition de la couverture végétale, ce qui favorise la lutte contre l'érosion des sols. Mais, dans des régions à forte concentration de forêts primaires, tel le Wallmapu, les conséquences sont plus violentes pour le paysage et la biodiversité. Ces plantations d'arbres à croissance rapide et fortes consommatrices d'eau, en acidifiant les sols, peuvent provoquer la disparition de la forêt primaire et des espèces liées à ce milieu. Dans ce cas, la politique de reboisement fragilise non seulement la biodiversité naturelle et donc la richesse de la faune et de la flore, mais aussi menace la diversité culturelle. Les différents agents intervenant dans le champ de la médecine traditionnelle (machi, curande- 
ros, meica...) se plaignent, en effet, de la disparition de nombreuses plantes médicinales dans les zones d'exploitation forestière. De la même manière, l'assèchement des sources d'eau et, donc, la disparition des ngen co (« les forces-maîtres de l'eau ") sont perçus comme un mauvais présage par les habitants des communautés qui les environnent. Ces préoccupations doivent pourtant cohabiter avec des nécessités quotidiennes de subsistance les contraignant à travailler pour les entreprises forestières.

Autour de la forêt et de son exploitation, on trouve aussi différents acteurs tels que les paysans bûcherons ou les ouvriers forestiers, les grandes entreprises d'exploitation du bois et de reboisement, des agences de l'État, du gouvernement local et national, et des ONG. La ressource la plus convoitée, la forêt originelle, se concentre au sud du pays dans une zone géographique à forte présence indigène ${ }^{20}$. Les terres des communautés sont les seules qui sont susceptibles d'être exploitées à des fins forestières. Aussi, l'État promeut, au sein même des communautés, par le biais de programmes de développement locaux et de " pertinence ethnique et culturelle ${ }^{21}$, des politiques contradictoires qui forcent la main à des milliers de paysans. Certes, dans le cadre de projets à court terme, on assiste à la distribution de plants d'arbres allogènes, de pins et d'eucalyptus, et la formation aux divers métiers forestiers (bûcherons, gardiens, chauffeurs), mais d'autres programmes de « développement avec identité » mettent en place des pépinières d'espèces natives dont les résultats ne se verront qu'après deux générations. Dans ces deux cas, la superficie ${ }^{22}$ restreinte de la plupart des terres constitue un problème majeur et non résolu, car elle empêche la polyculture et la mise en jachère. Pour lutter contre l'expansion des monocultures, diverses ONG (indigènes, nationales et internationales) se mobilisent, défendant la protection efficace et durable de cette forêt et des relations particulières entretenues par les communautés indigènes avec leur milieu.

\section{CELCO OU LA « GUERRE DE LA MER 》}

Le cas de la multinationale CELCO (Celulosa Arauco y Constitución) est représentatif des conflits provoqués par l'exploitation forestière à outrance. Cette entreprise a entamé la construction d'une usine de pâte à papier, en 1996, dans la zone côtière de Valdivia, à $900 \mathrm{~km}$ de la capitale. À cette époque comme aujourd'hui, CELCO prévoyait de se débarrasser des effluents (chlore entre autres) dans la mer, via une conduite longue d'une vingtaine de kilomètres débouchant dans la baie de Maiquillahue. Grâce à l'organisation et à la résistance de l'ensemble de la population locale, dont les Mapuche-Lafkenche (essentiellement des pêcheurs et leurs femmes), cela n'a pas pu se faire. L'usine a alors déversé ses déchets dans une rivière à proximité et quelques années plus tard, en 2004, l'effet fut catastrophique pour l'environnement, la flore et la faune avec 
notamment la diminution importante de la population du cygne à cou noir (Cygnus melancoryphus). CELCO est alors revenu à son idée initiale, à savoir le rejet des déchets en mer, s'obligeant à effectuer une étude d'impact et une série de relevés, ainsi que l'exige la Loi environnementale.

Les habitants de la baie de Maiquillahue, zone affectée au rejet des déchets, se sont alors organisés, tout comme sept ans auparavant, afin de s'opposer à la réalisation de cette étude. En mer, les pêcheurs artisans ont comme seul moyen d'action, pour se faire entendre, de s'organiser en flottilles. Ils empêchent les navires de CELCO, bien qu'escortés par l'armée, d'effectuer les relevés. Cette confrontation entre David et Goliath, entre des barques et un navire militaire, s'est à plusieurs reprises transformée en véritable " guerre de la mer », médiatisée à travers des images transmises par la télévision chilienne, mais également par de nombreux blogs et sites internet.

Les différentes autorités traditionnelles ont réalisé des rites, prières et invocations pour empêcher la pollution de leur source de vie, la lafken (« mer »); elles sont prêtes à tout pour sauver leur moyen de subsistance. Le conflit soulève ainsi des problèmes spirituels, symboliques, économiques et politiques. L'ambiance quasi belliqueuse et la tension quotidienne sont source d'angoisse et d'incertitude, surtout pour les femmes qui voient leurs maris prendre la mer pour défendre, à leurs risques et périls, leur ressource vitale.

Le conflit perdure aujourd'hui. L'entreprise a profité de son pouvoir économique pour " acheter » peu à peu différents dirigeants et pêcheurs. Elle leur a versé des sommes d'argent considérables en contrepartie de leur "collaboration " pour permettre la réalisation des relevés, ainsi que leur promesse de ne pas engager de poursuites en justice en cas de pollution de leur périmètre de pêche. Cette corruption a provoqué des confrontations à l'intérieur même de la communauté des pêcheurs et ceux qui résistent encore sont en majorité des Mapuche-Lafkenche.

\section{LA RECONFIGURATION DU PAYSAGE}

Cette résistance des populations concernées face aux grands projets s'inscrit dans le refus d'un modèle de développement à court terme qui modifie de façon radicale leur paysage et leur patrimoine matériel et immatériel. Bien que la reconfiguration du paysage par l'homme en territoire mapuche date de plusieurs siècles, on assiste ces dernières années à un changement radical et violent. C'est ainsi que les politiques de développement et d'exploitation des ressources naturelles, ressources de plus en plus rares, ont créé de vastes espaces de monocultures où auparavant il existait une riche diversité biologique. Cette exploitation intensive est liée à une forte demande à l'exportation. On assiste à l'affaiblissement du pouvoir de décision des États qui se sont déchargés de quelques-unes de leurs 
responsabilités, face aux pressions de politiques économiques internationales promouvant l'initiative privée.

Dans les communautés mapuche s'expriment encore aujourd'hui une diversité de savoirs et une forte imprégnation de pratiques culturelles ancrées dans la mémoire sociale, en rapport direct avec leur environnement. La diminution progressive de leurs ressources naturelles a eu pour conséquence la mise en péril de ces pratiques concernant leur milieu. C'est un sujet de discussion et de préoccupation des autorités traditionnelles qui voient désormais la naissance d'une esthétique paysagère néolibérale monotone. L'imposition d'un paysage standard, auquel ils ne participent qu'en tant que spectateurs, a provoqué, au fur et à mesure des années, diverses réactions chez les habitants des communautés.

\section{MOBILISATIONS, POLITIQUES PUBLIQUES ET CRIMINALISATION}

On l'a vu dans les différents exemples présentés, face au dynamisme d'un mouvement mapuche contemporain très hétérogène qui a su se faire entendre, notamment grâce aux nouvelles technologies, les autorités chiliennes et argentines n'ont pas pu rester indifférentes. Elles ont dû répondre d'une façon ou d'une autre à ces différents conflits soit en négociant, soit en proposant de nouvelles politiques en faveur des indigènes, soit en les judiciarisant. L'action de l'État n'a pas été uniforme, puisqu'elle a consisté en la combinaison de stratégies parfois contradictoires. Souvent les institutions chargées des affaires indigènes, la CONADI et l'INAI ${ }^{23}$, ont été sollicitées, mais elles ont été très rapidement dépassées étant donné les enjeux économiques et politiques de ces conflits. Les négociations qu'elles ont entreprises se sont souvent engagées avec des interlocuteurs qui n'étaient pas toujours reconnus de tous, au risque d'exacerber des divisions et de provoquer des confrontations au sein même des communautés. Les dirigeants les plus intransigeants ont été stigmatisés comme des Mapuche extrémistes, politisés et violents, justifiant ainsi la mise en place de modes de résolution de conflit plus musclés de la part des autorités.

Dans le cas du Chili, cette situation s'est illustrée par l'application d'une violence symbolique et physique envers les membres et dirigeants de communautés revendiquant des terres. Depuis dix ans, on compte ainsi, au bas mot, plus d'un demi-millier d'arrestations de personnes ${ }^{24}$, dont certaines sont aujourd'hui emprisonnées et condamnées à des peines allant jusqu'à dix ans de prison ${ }^{25}$. C'est dans ce même contexte répressif qu'une cinquantaine de personnes ont été gravement blessées par balle et qu'au moins deux jeunes Mapuche ont été abattus par la police chilienne. En Argentine, si la voie de la judiciarisation, c'est-à-dire la résolution des conflits par la force policière, puis devant les tribunaux, a été également suivie par les différents gouverneurs concernés, il ne semble pas qu'elle ait fait à ce jour autant de victimes ${ }^{26}$. Cette différence peut en partie s'expliquer 
par le fait que la figure de l'Indien dans le Sud de l'Argentine est souvent assimilée, par méconnaissance, à celle emblématique du gaucho, représentant l'« argentinité ». Cette forme de confusion entre l'Indien et la figure du gaucho confère au premier à la fois une relative invisibilité et une certaine respectabilité. Elle lui évite de n'être soumis qu'à des préjugés négatifs, contrairement à ce qui se passe au Chili où la représentation de l'indigène renvoie, encore aujourd'hui, à tout un imaginaire de l'Indien sauvage, violent et rebelle ${ }^{27}$.

La répression a également provoqué son lot de « contre-violences » de la part des Mapuche, quoique de moindre ampleur: barrages routiers, incendie de camions forestiers et de propriétés revendiquées. Ces mobilisations ont souvent été qualifiées d'actes terroristes ${ }^{28}$ par les autorités judiciaires. Convaincus que de telles mesures ne leur assuraient pas un procès équitable, certains dirigeants soupçonnés d'avoir commis ces actions de revendication ont préféré se réfugier en Argentine, bénéficiant de l'accueil et du soutien de «leurs frères et sœurs » de Puelmapu. Les fugitifs, les martyrs, tout comme les prisonniers politiques mapuche, deviennent des symboles pour un mouvement indigène de plus en plus diversifié et épars, composé d'intellectuels et d'artistes urbains, de communautés, d'organisations, de non-Mapuche, ainsi que d'un nouveau parti politique mapuche, Wallmapuwen (« compatriotes du pays mapuche »).

La renaissance politique et culturelle des Mapuche, observée ces quinze dernières années, s'est caractérisée par la capacité des communautés et organisations à jouer sur la tension entre opposition et négociation, impliquant un éventail de mobilisations et d'identifications. Les grandes réunions organisées chaque été, sur le modèle des protocoles d'antan, sont des espaces non seulement de rencontre, mais également de résistance et de revitalisation de la culture mapuche. Par leurs rituels, elles unissent des personnes venant de différentes zones, donnant un sens commun à une diversité d'expériences. Par l'extériorisation des conflits et les discussions politiques, ces rassemblements permettent aux participants de s'identifier et de se projeter en tant que membres d'un même peuple en quête de reconnaissance.

En ayant recours aux nouvelles technologies de l'information et de la communication et en allant devant les tribunaux nationaux et internationaux, les Mapuche ont réussi à médiatiser leurs revendications et à sensibiliser les sociétés civiles chilienne et argentine. À l'inverse, la criminalisation des revendications territoriales mapuche n'a pas eu les effets escomptés. Au lieu de démobiliser les communautés, cette répression a permis de médiatiser et de rendre plus visibles les conflits, mais surtout les violences commises envers leurs habitants. C'est dans ce contexte qu'un nouveau regard a pu être porté sur ces populations, leur culture et leur histoire, permettant des rapprochements au sein de sociétés fortement marquées par des préjugés raciaux. On a ainsi pu remarquer un regain d'intérêt de la part des Chiliens et des Argentins pour les manifestations culturelles mapuche, mais également pour leurs revendications politiques. ONG et institutions publi- 
ques ont ainsi dû transformer leur mode d'intervention et leur façon de se mettre en relation avec les communautés.

Cette préoccupation s'est illustrée par la multiplication de techniques d'ethno-gouvernementalité, imposant l'usage d'une nouvelle doxa accompagnée de son vocabulaire tel que le " capital social », le " développement avec identité » ou l'« ethnodéveloppement ». Des techniques qui permettent, bon gré, mal gré, la coexistence de politiques publiques destinées aux indigènes et l'exploitation à outrance de leurs ressources naturelles dont on ne peut pas négliger l'impact sur leur avenir. La défiguration et l'uniformisation des paysages du territoire des Mapuche ont pour effet de reformuler leur rapport à l'environnement et de naturaliser les inégalités sociales et historiques y existant.

Ces différentes formes de violence structurent la mémoire sociale des Mapuche, ceux des villes comme des campagnes, mais elles contribuent également à la formation de régimes de "s subjectivité mapuche » dépassant les catégories ethniques permettant aux sociétés créoles de redécouvrir leur histoire et leur origine. La surprenante internationalisation des revendications de ce mouvement marque l'émergence de nouvelles figures de représentants traditionnels, suscitant un grand intérêt en dehors du continent latino-américain. On a ainsi pu observer dans la foule du cortège parisien du $1^{\text {er }}$ mai dernier deux Mapuche marchant côte à côte. Tous deux arrivés de Wallmapu, ils venaient juste de faire connaissance. L'homme, habillé d'un jean et d'une chemise de bûcheron, un drapeau wenu foye à la main, était venu en Europe depuis sa communauté de Ngulumapu pour témoigner en tant qu'ex-prisonnier politique. La femme en habit traditionnel venait, quant à elle, de Puelmapu et effectuait une tournée européenne afin de défendre sa « terre-mère » contre les projets hydroélectriques à " capitaux européens ». Repartis dans leur communauté d'origine après plusieurs mois de tournée, leurs récits contribueront peut-être à forger, de part et d'autre de la Cordillère, les narrations modernes des " voyages en Worldmapu ».

\section{NOTES}

1. Étant donné que Mapuche, "gens de la terre », est un ethnonyme dont les origines ne sont pas latines, il ne sera pas accordé au pluriel et portera une majuscule, sauf lorsqu'il est utilisé comme adjectif. L'écriture de la langue mapuche, le mapudungun, étant un sujet de controverses car il existe plusieurs graphies, nous privilégierons l'usage de l'écriture considérée comme la plus simple à écrire, la " graphie unifiée ». Quant aux noms de personnes et d'organisations en mapudungun, ils garderont la graphie que celles-ci ont décidé d'adopter.

2. Pour le Chili : Instituto Nacional de Estadística (INE), 2005, Estadisticas sociales de pueblos indigenas en Chile - Censo 2002, Santiago ; pour l'Argentine : INDEC, 2005, Encuesta complementaria de pueblos indigenas (ECPI) 2004-2005 - complementaria del censo nacional de población, hogares y viviendas, 2001, Buenos Aires.

3. Delrio Walter, 2005, Memorias de expropiación. Sometimiento e incorporación indigena en Patagonia (1872-1943), Editorial de la Universidad de Quilmes, Buenos Aires, pp. 38-39. 
4. Ancán José, 2000, « Los napülkafe, viajeros del wallmapu, en el antiguo paisaje mapuche », in Carlos Aldunate (éd.), Voces mapuche. Mapuche dungu, Editorial Museo Chileno de Arte Precolombino, Santiago, pp. 99-139.

5. Au Chili, la loi régulant la venue des colons étrangers établissait que chaque famille venant d'Europe et des États-Unis devait recevoir un minimum de 150 hectares, auquel s'ajoutait la moitié de cette superficie pour chaque enfant majeur de 10 ans. Par ailleurs, l'État leur octroyait le transport gratuit jusqu'au Chili, des clous, des planches, une paire d'animaux de trait, un araire, une charrette...

6. Le quotidien de Temuco, El Diario Austral, évoquait ainsi l'exode des Mapuche de l'Araucanie vers l'Argentine, en 1918 : «Tristes, silencieux, résignés et abattus, formant une caravane afligée et immense, les quelques Araucans restant dans la zone australe de notre pays s'en vont en exode pénible. Et c'est honteux de le dire, mais ils n'abandonnent pas leurs terres car celles-ci sont stériles. Ils s'en vont en Argentine à la recherche d'une tranquillité qu'ils méritent et qu'on leur a refusée, ils sont à la recherche de protection, de justice. Ils fuient des étrangers avares et criminels qui leur ont usurpé leurs terrains » (" Los mapuches emigran », El Diario Austral de Temuco, $\mathrm{n}^{\circ}$ 694, miércoles 3 de febrero de 1918, p. 2, cité in Jorge Pinto Rodríguez, 2007, «Expansión económica y conflicto mapuche. La Araucanía, 1900-1940 ", Revista de Historia Social y de las Mentalidades, XI (1), pp. 9-34).

7. Guillaume Boccara, 2006, "The brighter side of the indigenous renaissance (Part. 1) ", Nuevo Mundo. Mundos Nuevos, Debates (en ligne : http://nuevomundo.revues.org/index2405.html, mis en ligne le 16 juin 2006, consulté le 4 août 2008).

8. Azkintuwe ( Le Mirador »), fondé en 2003, est le premier journal mensuel mapuche couvrant l'actualité et diffusé en Ngulumapu et Puelmapu (en ligne, http://www.azkintuwe.org/, mis en ligne le 29 novembre 2004, consulté le 23 septembre 2008).

9. « $\mathrm{Coz} \mathrm{Coz}$ » désigne le lieu du Parlamento, près de Panguipulli, $\mathrm{X}^{\mathrm{e}}$ région, Chili. Les organisations contemporaines qui ont remis à jour cet événement ont préféré utiliser le graphème de Raguileo, le seul créé par un Mapuche et réputé le plus fidèle à la prononciation du mapudungun, pour l'écrire "Koz Koz ». Nous différencierons ainsi le Parlamento de Coz Coz de 1907 du Parlamento de Koz Koz de 2007.

10. Diaz Meza Aurelio, 2007 [1907], Parlamento de Coz Coz, Comisión de Salud Intercultural/Corporación Municipal de Panguipulli, Panguipulli, Chili, p. 15.

11. Maison traditionnelle faite d'une ossature de bois et recouverte de paille.

12. Marimán Quemenado Pablo, 2002, "Recuperar lo propio será siempre fecundo », in Roberto Morales Urra (éd.), Territorialidad mapuche en el siglo xx, Instituto de Estudios Indígenas, Temuco, pp. $51-121$.

13. Toledo Víctor, 2006, El pueblo mapuche, derechos colectivos y territorio: desafios para la sustentabilidad democrática, Ed. Chile Sustentable, LOM, Santiago.

14. En ligne, http://www.am800wajzugun.org.ar, mis en ligne en 2006, consulté le 22 juin 2008.

15. L'importance de leur population (1 200 personnes) et leur superficie territoriale (environ 14000 hectares) en font les communautés les plus peuplées et les plus étendues de Puelmapu.

16. Cité par la Comisión de Prensa de Wallmapuiven, in « Oposición al proyecto Aeropuerto llegará hasta el Congreso ", (en ligne, http://www.wallmapuwen.cl/noti_abrill6.htm, mis en ligne le 17 avril 2007, consulté le 4 août 2008).

17. Forte de ces différentes constatations, la Fédération internationale des Droits de l'Homme a pu avancer la conclusion que le projet était " ethnocidaire ".

18. Voir María José Reis, Juan Carlos Radovich et al. (éds), 2005, Disputas territoriales y conflictos interétnicos en Brasil y Argentina, Ed. Ferreyra, Córdoba.

19. En ligne, http://www.patagoniasinrepresas.cl/, mis en ligne en décembre 2007, consulté le 22 juillet 2008 .

20. Barrué-Pastor Monique, 2004, Forêts et développement au Chili. Indianité mapuche et mondialisation, Presses universitaires du Mirail, Toulouse.

21. La " pertinence » ethnique et culturelle a été le maître-mot des programmes de développement indigène mis en place ces dernières années par le gouvernement chilien et la Banque interaméricaine de 
Développement (en ligne, http://www.origenes.cl, mis en ligne en 2001, consulté le 29 juillet 2008). Le terme est également utilisé comme argument dans les rapports faisant la promotion de certains " megaprojets », afin de minimiser l'impact de ceux-ci.

22. Dans le cas des communautés de Ngulumapu, on l'estime à une moyenne de 5 hectares par personne, mais avec de grandes disparités, tant au niveau de la qualité des terres que de leur superficie, selon les zones géographiques.

23. La Corporación Nacional de Desarrollo Indigena (CONADI), créée en 1993 en application de la Loi indigène, a pour fonction la protection, la promotion et le développement des indigènes au Chili. L'Instituto Nacional de Asuntos Indigenas (INAI), créé par décret en 1985, a commencé à fonctionner dans les années 1990 afin de « créer les canaux interculturels pour la mise en place des droits des Peuples Indigènes consacrés par la Constitution argentine de 1994 ».

24. Il faut remarquer que la répression ne concerne pas seulement les Mapuche, mais également les personnes qui soutiennent leurs revendications, comme dans le cas de la réalisatrice Elena Varela qui a été arrêtée au mois de mai 2008, son matériel audiovisuel saisi, pour avoir voulu rendre compte de la violence exercée envers les communautés (en ligne, http://porlalibertadexpresion.entodaspartes.net/, mis en ligne le 3 juillet 2008, consulté le 18 juillet 2008).

25. On dénombre actuellement une vingtaine de « prisonniers politiques mapuche » dans les geôles du sud du Chili. Cette autodénomination, apparue au début des années 2000, est couramment utilisée pour qualifier les personnes, d'origine mapuche ou non, détenues dans le contexte des conflits territoriaux.

26. Pour avoir des informations sur la situation en Puelmapu, on peut notamment consulter : en ligne, http://www.avkinpivkemapu.com.ar/, mis en ligne en juillet 2004, consulté le 7 août 2008, et http://www.lofdigital.org.ar/, mis en ligne le 15 novembre 2005, consulté le 7 août 2008.

27. Malgré une image relativement moderne du Mapuche diffusée dans les médias chiliens, on peut encore trouver des opinions émises par des personnalités régionales le qualifiant de «malin, louche, déloyal et abuseur ", " prédateur ", " vivant de ce que lui apporte la nature, sans capacité intellectuelle, ni volonté ", et suggérant la nécessité de le "recycler » et de mener " une nouvelle pacification de l'Araucanie ".

28. Depuis le début des années 2000, la Loi anti-terroriste (18.314) édietée sous la dictature (1984) a été utilisée au moins à six reprises et exclusivement dans le cadre du conflit mapuche. Elle s'est soldée par la condamnation d'une dizaine de personnes à des peines de cinq à dix années de prison, pour des menaces ou des incendies de propriétés forestières.

Ana Guevara, EHESS/LAS, Collège de France [guevara@ehess.fr] Fabien Le Bonniec, EHESS/IRIS, Universidad de Chile [wenuy@yahoo.fr] 